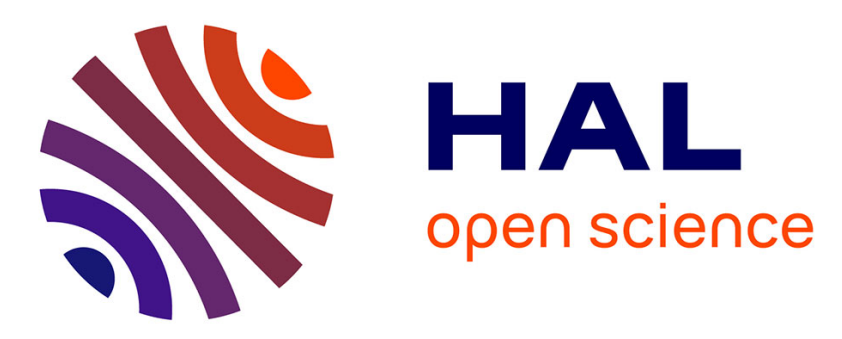

\title{
Streaming constrained binary logistic regression with online standardized data
}

Benoît Lalloué, Jean-Marie Monnez, Eliane Albuisson

\section{To cite this version:}

Benoît Lalloué, Jean-Marie Monnez, Eliane Albuisson. Streaming constrained binary logistic regression with online standardized data. Journal of Applied Statistics, 2022, 49 (6), pp.1519-1539. 10.1080/02664763.2020.1870672 . hal-02156324v4

\section{HAL Id: hal-02156324 \\ https://hal.science/hal-02156324v4}

Submitted on 7 Jan 2021

HAL is a multi-disciplinary open access archive for the deposit and dissemination of scientific research documents, whether they are published or not. The documents may come from teaching and research institutions in France or abroad, or from public or private research centers.
L'archive ouverte pluridisciplinaire HAL, est destinée au dépôt et à la diffusion de documents scientifiques de niveau recherche, publiés ou non, émanant des établissements d'enseignement et de recherche français ou étrangers, des laboratoires publics ou privés. 


\title{
ORIGINAL RESEARCH ARTICLE
}

\section{Streaming constrained binary logistic regression with online standardized data.}

\author{
Benoît Lalloué $^{\mathrm{a}, \mathrm{b},{ }^{*}}$, Jean-Marie Monnez ${ }^{\mathrm{a}, \mathrm{b}, \dagger}$ and Eliane Albuisson ${ }^{\mathrm{c}, \mathrm{d}, \mathrm{e}, \ddagger}$ \\ ${ }^{a}$ Université de Lorraine, CNRS, Inria (Project-team BIGS), IECL (Institut Elie Cartan de Lorraine), \\ BP239, F-54506 Vandœuvre-lès-Nancy, France \\ ${ }^{\mathrm{b}}$ CHRU Nancy, INSERM, Université de Lorraine, CIC Plurithématique, F-54000 Nancy, \\ France \\ ${ }^{c}$ Université de Lorraine, CNRS, IECL, F-54000 Nancy, France \\ ${ }^{\mathrm{d} B I O B A S E}$, Pôle S2R, CHRU de Nancy, Vandœuvre-lès-Nancy, France \\ ' Faculté de Médecine, InSciDenS, Vandœuvre-lès-Nancy, France
}

\section{ARTICLE HISTORY}

Compiled October 13, 2020

\begin{abstract}
Online learning is a method for analyzing very large datasets ("big data") as well as data streams. In this article, we consider the case of constrained binary logistic regression and show the interest of using processes with an online standardization of the data, in particular to avoid numerical explosions or to allow the use of shrinkage methods. We prove the almost sure convergence of such a process and propose using a piecewise constant step-size such that the latter does not decrease too quickly and does not reduce the speed of convergence. We compare twenty-four stochastic approximation processes with raw or online standardized data on five real or simulated data sets. Results show that, unlike processes with raw data, processes with online standardized data can prevent numerical explosions and yield the best results.
\end{abstract}

\section{KEYWORDS}

Big data; Data stream; Logistic regression; Online learning; Stochastic approximation; Stochastic gradient

\section{Introduction}

\subsection{Background}

Data stream online analysis concerns data that arrive continuously such as process control data, web data, telecommunication data, medical data or financial data. Online learning, which proceeds in successive steps, the results of which are being updated at each step taking into account a batch of new data, is particularly adapted to data streams. For observations arriving sequentially, recursive stochastic algorithms can be used to estimate, for instance, parameters of a linear regression model $[8,10]$, principal components of a factorial analysis [18] or centers of classes in non-hierarchical clustering [6], whose estimations are updated by each new arriving data batch. When

\footnotetext{
* benoit.lalloue@univ-lorraine.fr ; https://orcid.org/0000-0002-7433-9678

† jean-marie.monnez@univ-lorraine.fr

$\ddagger$ eliane.albuisson@univ-lorraine.fr
} 
using such recursive processes, it is not necessary to store the data and, due to the relative simplicity of the computation involved, a much greater number of data can be taken into account than with classical methods during the same amount of time. Therefore, recursive algorithms can also profitably be used for very large datasets (by randomly drawing a data batch from the dataset at each step) when the capacities of statistical learning methods are potentially limited by computing time. Batch gradient and stochastic gradient methods are presented and compared in [4] and reviewed in [3].

When using a stochastic gradient process, a numerical explosion can be encountered [19]. To avoid such phenomenon, methods of gradient variance reduction [3, 13], such as gradient clipping [19], can be used. The idea underpinning gradient clipping is to limit the norm of the gradient to a maximum number called threshold. This number must be chosen and a poor choice of threshold can affect computing speed. In our approach, the limitation of the gradient is implicitly obtained by online standardization of the data: each continuous variable is standardized with respect to the estimations at the current step of its expectation and its standard deviation computed online. Indeed, in the case of a data stream, the mathematical expectation and the variance of each variable are a priori unknown and the usual offline standardization cannot be performed. This may also be an issue when using a shrinkage method such as LASSO or ridge, which first necessitates standardizing the explanatory variables. Again, it is not possible to perform the offline standardization in the case of a data stream and an online process can be used, with a projection at each step on the convex set defined by the constraint on the parameters of the regression function. More generally this type of process can be used for any convex set, for example if it is imposed that the parameters associated with the explanatory variables are positive. Finally, we can consider a case where the expectations and the variances of the explanatory variables depend on the step $n$ or on the values of controlled variables and a regression model with standardized explanatory variables is defined. Assuming that we can estimate online the expectation and the variance of these variables, we can also use the same type of process to estimate the parameters of the regression function.

In a previous study [10] addressing sequential least square multidimensional linear regression using a stochastic approximation process, we proved the convergence of three processes with online standardized data instead of raw data, discussed the advantages of this approach compared to other methods, and experimentally showed that processes with online standardized data were superior to processes with raw data. In the present study, we use a similar approach in the case of constrained binary logistic regression, using a stochastic gradient process with online standardization of the data. Herein, the second Lyapounov method ([14], p.9) is used in the proof of convergence, the additional problem being that the expectations and variances of the explanatory variables are unknown but replaced by convergent online estimations, as in the case of sequential linear regression [10]. We consider an averaged stochastic gradient process: intuitively, when the algorithm is not too distant from the solution, averaging allows decreasing the variability of the initial algorithm, which can oscillate around the true solution, and thereby improve its performance [21][6].

Since a suitable choice of step-size is often crucial for obtaining good performances for stochastic gradient processes, we examined various choices of step-size. Bach and Moulines [2] already showed that a constant step-size averaged stochastic gradient process does not converge to the true value of the parameter (because the gradient of the loss function is not linear in the case of logistic regression) and alternatively defined other processes with a Newton-approximation scheme. However, Bach [1] suggested 
using a decreasing piecewise constant step-size, in order that the step-size does not decrease too quickly and does not reduce the speed of convergence, which we test in the present experiments and compare the latter to a more classical decreasing step-size.

Subsection 1.2 is devoted to the formulation of the problem, which is a problem of Stochastic Optimization of the expectation $F(x, a)$ of a random variable $Y(x, a)$ depending on an unknown parameter $a$ which is estimated online along with the stochastic gradient process, Subsection 1.3 to a comparison with other recent formulations such as Stochastic Compositional Optimization [23] and Conditional Stochastic Optimization [12], Subsection 2.1 to a definition and to a theorem of almost sure convergence of the stochastic gradient process, Subsection 2.2 to a comparison with the Stochastic Compositional Gradient Descent algorithm [23] as well as some possible extensions of our work, while Section 3 is devoted to the results of experiments where processes with raw data are compared to processes with online standardized data. The article ends with a conclusion (Section 4) and two appendices: Appendix A contains the proof of the theorem and Appendix B features additional experimental results.

\subsection{Formulation of the problem}

Let $A^{\prime}$ be the transpose of a matrix $A$. The abbreviation a.s. stands for almost surely.

Consider a data stream and assume that the observed data are realizations of a random vector $\left(R^{1}, \ldots, R^{p}, S\right)$ in $\mathbb{R}^{p} \times\{0,1\}$. Let $R$ be the random column vector $\left(R^{1} \ldots R^{p} 1\right)^{\prime}, m=\left(E\left[R^{1}\right] \ldots E\left[R^{p}\right] 0\right)^{\prime}, R^{c}=R-m\left(r^{c}\right.$ a realization of $\left.R^{c}\right), \sigma^{k}$ the standard deviation of $R^{k}(k=1, \ldots, p), \Gamma$ the diagonal $(p+1, p+1)$ matrix with diagonal elements $\frac{1}{\sigma^{1}}, \ldots, \frac{1}{\sigma^{p}}, 1$ (taking by convention $\sigma^{k}=1$ for a categorical variable), $Z=\Gamma R^{c}$ the vector $R$ whose continuous components are standardized $\left(z=\Gamma r^{c}\right.$ a realization of $Z$ ) and $\theta=\left(\theta^{1} \ldots \theta^{p} \theta^{p+1}\right)^{\prime}$ a column vector of real parameters.

Consider the logistic model with standardized covariates:

$$
\begin{gathered}
P(S=s \mid Z=z)=f(s ; z, \theta)=\left(\frac{e^{z^{\prime} \theta}}{1+e^{z^{\prime} \theta}}\right)^{s}\left(\frac{1}{1+e^{z^{\prime} \theta}}\right)^{1-s}=\frac{e^{z^{\prime} \theta s}}{1+e^{z^{\prime} \theta}} . \\
E[S \mid Z]=h\left(Z^{\prime} \theta\right) \text { with } h(u)=\frac{e^{u}}{1+e^{u}}=\frac{1}{1+e^{-u}} .
\end{gathered}
$$

Remark 1. Let $m^{j}=E\left[R^{j}\right], j=1, \ldots, p$. Note that if $\theta_{0}$ is the column vector of the parameters of the logistic regression function of $S$ with respect to $R$, then $f(s ; z, \theta)=$ $f_{0}\left(s ; r, \theta_{0}\right)=\frac{e^{r^{\prime} \theta_{0} s}}{1+e^{r^{\prime} \theta_{0}}}$, with for $j=1, \ldots, p$ :

$$
\theta_{0}^{j}=\frac{\theta^{j}}{\sigma^{j}}, \theta_{0}^{p+1}=\theta^{p+1}-\left(\sum_{j=1}^{p} m^{j} \frac{\theta^{j}}{\sigma^{j}}\right) \Leftrightarrow \theta_{0}=\left(\begin{array}{cccc}
\frac{1}{\sigma^{1}} & & & \\
& \ddots & & \\
& & \frac{1}{\sigma^{p}} & \\
-\frac{m^{1}}{\sigma^{1}} & \cdots & -\frac{m^{p}}{\sigma^{p}} & 1
\end{array}\right) \theta
$$

Define the loss function $-\ln f(s ; z, x)=\ln \frac{1+e^{z^{\prime} x}}{e^{z^{\prime} x s}}$. The cost function

$$
F(x)=-E[\ln f(S ; Z, x)]=E\left[\ln \frac{1+e^{Z^{\prime} x}}{e^{Z^{\prime} x S}}\right]=E\left[-Z^{\prime} x S+\ln \left(1+e^{Z^{\prime} x}\right)\right]
$$

has $\theta$ for unique minimizer since $F$ is a convex function with positive Hessian 


$$
F^{\prime \prime}(x)=E\left[Z Z^{\prime} \frac{e^{Z^{\prime} x}}{\left(1+e^{Z^{\prime} x}\right)^{2}}\right] .
$$

$\theta$ is the unique solution of:

$$
F^{\prime}(x)=E\left[-Z S+\frac{Z e^{Z^{\prime} x}}{1+e^{Z^{\prime} x}}\right]=E\left[Z\left(h\left(Z^{\prime} x\right)-S\right)\right]=0 .
$$

The purpose of this study is to recursively estimate $\theta$ using a stochastic gradient algorithm with online standardized data.

\subsection{Comparison with other formulations}

Let $R^{2}$ denote the random column $p$-vector $\left(\left(R^{1}\right)^{2} \ldots\left(R^{p}\right)^{2}\right)^{\prime}$ and $g(R)$ denote the random column $2 p+1$-vector $\left(R^{\prime} R^{2 \prime}\right)^{\prime}$.

The diagonal matrix $\Gamma$ is a function of $E[R]$ and $E\left[R^{2}\right]$, thus of $E[g(R)]: \Gamma=$ $C(E[g(R)])$. Moreover $E[R]=A E[g(R)]$, with $A=\left(I_{p+1}(0)\right), I_{p+1}$ the identity matrix of order $p+1$ and $(0)$ the null $(p+1, p)$ matrix. We can then write $F(x)=$ $F(x, E[g(R)])$ as the expectation of a function $f$ of $x$ parametrized by $E[g(R)], R, S$ :

$$
\begin{aligned}
F(x) & =F(x, E[g(R)])=E\left[\ln \frac{1+\exp \left((R-A E[g(R)])^{\prime} C(E[g(R)]) x\right)}{\exp \left(S(R-A E[g(R)])^{\prime} C(E[g(R)]) x\right)}\right] \\
& =E[f(x, E[g(R)] ; R, S)] .
\end{aligned}
$$

A. The minimization of $F(x)$ is a problem of Stochastic Optimization of the expectation of a random variable $Y(x, a)$ that depends on an unknown parameter $a=E[g(R)]$ which is estimated online by iterative averaging along with the solution $\theta$. We have dealt with this type of problem in other settings, for example in a streaming multiple factor analysis of a random vector $Z$ [15] or in a streaming generalized canonical correlation analysis [17] where unknown elements such as an expectation or a covariance matrix or a metric are estimated online along with the principal components. We extended this approach in [16] to the case of principal component analysis of a random vector with a time-varying expectation. We present in Subsection 2.2 the same type of extension.

B. In [7], the authors studied the minimization on a compact set of composite risk functions, in particular

$$
F(x)=E_{V}\left[f\left(x, E_{V} g(x ; V) ; V\right)\right] .
$$

Formulation (6) can be considered as a particular case of (7) with $g$ not depending on $x$. However, the authors only established a central limit theorem for the empirical estimator minimizing the empirical risk obtained by replacing the expectations in (7) by empirical means over a finite sample. Here, we solve the minimization of $F(x)(6)$ directly by using a stochastic approximation process including the sequential estimation of $E[g(R)]$ by iterative averaging. 
C. The aim of Stochastic Compositional Optimization (SCO [23], [11], [25] for multilevel compositional optimization, and references therein) is to minimize the composition of two expected-value functions:

$$
F(x)=E_{V}\left[f\left(E_{W} g(x ; W) ; V\right)\right]
$$

Taking $V=(R, S), W=R$ and $g(x ; R)=\left(x^{\prime}, g(R)^{\prime}\right)^{\prime}$, this formulation is formally the same as in equation (6). Thus formulation (6) could be considered as a particular case of (8) and the convergence results of SCO applied to the problem (6). However, we show in Subsection 2.2 that a larger choice of step-sizes than in [23] is allowed by our convergence analysis using a classical method of stochastic approximation.

D. The aim of Conditional Stochastic Optimization (CSO, [12]) is to minimize

$$
F(x)=E_{V}\left[f\left(E_{W / V} g(x ; V, W) ; V\right)\right] .
$$

This formulation does not apply to the present case since there is no conditional expectation in formulation (6).

In conclusion, the sequential minimization of $F(x)$ in (6) is a stochastic approximation problem involving a stochastic gradient process and requiring the simultaneous online estimation of the unknown expectation of $g(R)$.

\section{Approach: Definition of a stochastic gradient process}

\subsection{Definition and convergence}

Let $\left(\left(R_{n}^{1}, \ldots, R_{n}^{p}, S_{n}\right), n \geqslant 1\right)$ be an i.i.d. sample of $\left(R^{1}, \ldots, R^{p}, S\right)$ and, for $n \geqslant 1, R_{n}=$ $\left(R_{n}^{1} \ldots R_{n}^{p} 1\right)^{\prime}, R_{n}^{c}=R_{n}-m$ and $Z_{n}=\Gamma R_{n}^{c}$. For $k=1, \ldots, p$, let $\bar{R}_{n}^{k}$ be the mean of the sample $\left(R_{1}^{k}, \ldots, R_{n}^{k}\right)$ of $R^{k}$ and $\left(V_{n}^{k}\right)^{2}=\frac{1}{n} \sum_{i=1}^{n}\left(R_{i}^{k}-\overline{R_{n}^{k}}\right)^{2}$ its variance (both recursively computed), $\bar{R}_{n}=\left(\overline{R_{n}^{1}} \ldots \overline{R_{n}^{p}} 0\right)^{\prime}$ and $\Gamma_{n}$ the $(p+1, p+1)$ diagonal matrix with diagonal elements $\frac{1}{\sqrt{\frac{n}{n-1}} V_{n}^{1}}, \ldots, \frac{1}{\sqrt{\frac{n}{n-1}} V_{n}^{p}}, 1$.

Assume that $m_{n}$ observations $\left(R_{i}, S_{i}\right)$ are taken into account at step $n$ of the following defined process. Let $\mu_{n}=\sum_{i=1}^{n} m_{i}, I_{n}=\left\{\mu_{n-1}+1, \ldots, \mu_{n}\right\}$ be the set of indices of the observations taken into account at step $n, \widehat{R}_{n}=\bar{R}_{\mu_{n}}, \widehat{\Gamma}_{n}=\Gamma_{\mu_{n}}$ and for $j \in I_{n}$ :

$$
\widetilde{Z}_{j}=\widehat{\Gamma}_{n-1}\left(R_{j}-\widehat{R}_{n-1}\right)=\widehat{\Gamma}_{n-1}\left(R_{j}^{c}-\widehat{R}_{n-1}^{c}\right) \text { with } \widehat{R}_{n-1}^{c}=\widehat{R}_{n-1}-m
$$

For $k=1, \ldots, p$, each component $R_{j}^{k}$ of $R_{j}$ is pseudo-standardized with respect to the empirical mean $\widehat{R}_{n-1}^{k}$ and to the empirical estimation of $\sigma^{k}, \sqrt{\frac{\mu_{n-1}}{\mu_{n-1}-1}} V_{\mu_{n-1}}^{k}$. Note that all data up to step $n-1$ are used to estimate $m$ and $\Gamma$ at step $n$ by $\hat{R}_{n-1}$ and $\hat{\Gamma}_{n-1}$ respectively, which are recursively computed.

Assume that $\theta$ is constrained to belong to a convex subset $K$ of $\mathbb{R}^{p+1}$ (if there is no constraint, $\left.K=\mathbb{R}^{p+1}\right)$. Let $\Pi$ be the projection operator on $K$. Recursively define the stochastic approximation process $\left(X_{n}, n \geq 1\right)$ and the averaged process $\left(\bar{X}_{n}, n \geq 1\right)$ 
in $\mathbb{R}^{p+1}$ such that:

$$
\begin{aligned}
& X_{n+1}=\Pi\left(X_{n}-a_{n} \frac{1}{m_{n}} \sum_{j \in I_{n}} \widetilde{Z}_{j}\left(h\left(\widetilde{Z}_{j}^{\prime} X_{n}\right)-S_{j}\right)\right), \\
& \bar{X}_{n+1}=\frac{1}{n+1} \sum_{i=1}^{n+1} X_{i}=\bar{X}_{n}-\frac{1}{n+1}\left(\bar{X}_{n}-X_{n+1}\right) .
\end{aligned}
$$

Remark 2. The use of the projection operator $\Pi$ is only necessary if $\theta$ is constrained to belong to a convex set $\mathrm{K}$, for example when using a shrinkage method. It is not the case if we wish only to avoid a numerical explosion, as in the experiments conducted in Section 3.

Assume:

(H1a) There is no affine relation between the components of $R$.

(H1b) The moments of order 4 of $R$ exist.

(H2) $a_{n}>0, \sum_{n=1}^{\infty} a_{n}=\infty, \sum_{n=1}^{\infty} \frac{a_{n}}{\sqrt{n}}<\infty, \sum_{n=1}^{\infty} a_{n}^{2}<\infty$.

Theorem 2.1. Under $H 1 a, b$ and $H 2,\left(X_{n}\right)$ and $\left(\bar{X}_{n}\right)$ converge almost surely to $\theta$.

The proof using the second Lyapounov method ([14], p.9), also valid in the case of linear regression, is shown in Appendix A.

\subsection{Discussion and possible extensions}

A. The SCGD (Stochastic Compositional Gradient Descent) algorithm [23] used to solve the minimization of $F(x)$ in (8) is the composition of a stochastic gradient descent algorithm $\left(X_{n}\right)$ with step-size $\left(\alpha_{n}\right)$ and of an iterative weighted algorithm $\left(Y_{n}\right)$ with step-size $\left(\beta_{n}\right)$ such that:

$$
\begin{aligned}
X_{n+1} & =\Pi\left(X_{n}-\alpha_{n} \nabla g\left(X_{n} ; W_{n}\right) \nabla f\left(Y_{n+1} ; V_{n}\right)\right), \\
Y_{n+1} & =\left(1-\beta_{n}\right) Y_{n}+\beta_{n} g\left(X_{n} ; W_{n}\right),
\end{aligned}
$$

$\Pi$ being the projection operator on a closed convex set, $\left(V_{n}, W_{n}\right)$ an i.i.d. observation of $(V, W),\left(X_{n}\right)$ depending on $\left(Y_{n}\right)$ and $\left(Y_{n}\right)$ on $\left(X_{n}\right), \alpha_{n}$ and $\beta_{n}$ verifying the assumptions

$$
\sum_{n=1}^{\infty} \alpha_{n}=\infty, \sum_{n=1}^{\infty} \beta_{n}=\infty, \sum_{n=1}^{\infty}\left(\alpha_{n}^{2}+\beta_{n}^{2}+\frac{\alpha_{n}^{2}}{\beta_{n}}\right)<\infty
$$

to ensure the a.s. convergence of the algorithm $\left(X_{n}\right)$ ([23], Theorem 1). It requires that the algorithm $\left(X_{n}\right)$ with step-size $\left(\alpha_{n}\right)$ must be slower than the algorithm $\left(Y_{n}\right)$ with step-size $\left(\beta_{n}\right)$, which decreases the speed of convergence and creates practical difficulties according to [11].

B. Consider the algorithm $\left(X_{n}\right)$ defined in equation (11). To estimate $E\left[R^{j}\right]$ and $\operatorname{Var}\left[R^{j}\right]$ for $j=1, \ldots, p$, we estimate $E[g(R)]$ whose unknown components are $E\left[R^{j}\right]$ and $E\left[\left(R^{j}\right)^{2}\right], j=1, \ldots, p$, by iterative averaging, introducing at each step $n$ a minibatch of $m_{n}$ observations $R_{k}, k \in I_{n}$, and estimating $E[g(R)]$ at step $n$ by $M_{n}$ such 
that

$$
\begin{aligned}
M_{n+1} & =\left(1-\beta_{n}\right) M_{n}+\beta_{n} \frac{1}{m_{n}} \sum_{i \in I_{n}} g\left(R_{i}\right), M_{1}=0, \text { with } \beta_{n}=\frac{m_{n}}{\mu_{n}}, \text { thus } \\
M_{n+1} & =\frac{1}{\mu_{n}} \sum_{i=1}^{\mu_{n}} g\left(R_{i}\right) .
\end{aligned}
$$

Then $\widehat{R}_{n-1}=\left(M_{n}^{1} \ldots M_{n}^{p} 1\right)$ and $\widehat{V}_{n-1}^{i}=M_{n}^{p+1+i}-\left(M_{n}^{i}\right)^{2}, i=1, \ldots, p$. Thus the algorithm $\left(X_{n}\right)$ defined by equation (11) depends on $\left(M_{n}\right)$.

C. Consider the case where $m_{n}=1$ for all $n$ and compare (11) and (12) with SCGD defined by (13) and (14) taking $\alpha_{n}=a_{n}$ and $\beta_{n}=\frac{m_{n}}{\mu_{n}}=\frac{1}{n}$.

According to definition (14) of $\left(Y_{n}\right)$, as $g(x ; R)=\left(\begin{array}{c}x \\ g(R)\end{array}\right)$ and $E[g(x ; R)]=\left(\begin{array}{c}x \\ E[g(R)]\end{array}\right)$, we have $Y_{n}=\left(\begin{array}{c}Y_{n}^{1} \\ Y_{n}^{2}\end{array}\right)$ and:

a) $Y_{n+1}^{1}=\left(1-\beta_{n}\right) Y_{n}^{1}+\beta_{n} X_{n}=\frac{1}{n} \sum_{i=1}^{n} X_{i}=\bar{X}_{n}$, with $\mathrm{Y}_{1}^{1}=0$;

b) $Y_{n+1}^{2}=\left(1-\beta_{n}\right) Y_{n}^{2}+\beta_{n} g\left(R_{n}\right)$, thus $Y_{n+1}^{2}=M_{n+1}$, with $Y_{1}^{2}=0$;

c) $X_{n+1}=\Pi\left(X_{n}-a_{n} \widetilde{Z}_{n}\left(h\left(\widetilde{Z}_{n}^{\prime} \bar{X}_{n}\right)-S_{n}\right)\right)$;

thus, SCGD is different from the algorithm defined by (11) and (12) as $h\left(\widetilde{Z}_{n}^{\prime} X_{n}\right)$ in (11) is replaced by $h\left(\widetilde{Z}_{n}^{\prime} \bar{X}_{n}\right)$;

d) if we use in definition (11) of $\left(X_{n}\right)$ with $m_{n}=1$, the step-size $a_{n}=\frac{1}{n^{\alpha}}, \frac{1}{2}<\alpha \leq 1$, and take $\beta_{n}=\frac{1}{n}$, then $\frac{a_{n}^{2}}{\beta_{n}}=\frac{1}{n^{2 \alpha-1}} \geqslant \frac{1}{n}$, thus (15) is not verified; however, $\left(a_{n}\right)$ verifies the assumption $\sum_{n=1}^{\infty} a_{n}=\infty, \sum_{n=1}^{\infty} a_{n}^{2}<\infty, \sum_{n=1}^{\infty} \frac{a_{n}}{\sqrt{n}}<\infty$.

D. A more general step-size $\beta_{n}$ could be taken and, in the definition of $\widetilde{Z}_{j}, j \in I_{n}$, $\widehat{R}_{n-1}$ could be replaced by $\left(M_{n}^{1} \ldots M_{n}^{p} 1\right)^{\prime}$ and $\widehat{\Gamma}_{n-1}$ by a $(p+1, p+1)$ diagonal matrix with diagonal elements $\frac{1}{\sqrt{M_{n}^{p+1+i}-\left(M_{n}^{i}\right)^{2}}}, i=1, \ldots, p$, and 1 . Then it can be proved that $\left(M_{n}\right)$ converges a.s. to $E[g(R)]$ and $\left(X_{n}\right)$ defined by (11) to $\theta$ under the following assumptions on $\left(\beta_{n}\right)$ :

$$
\begin{aligned}
& \beta_{n}>0, \frac{\beta_{n}}{\beta_{n+1}} \leq 1+\gamma \beta_{n}+\gamma_{n}+o\left(\beta_{n}\right), \gamma<2, \gamma_{n} \geq 0, \sum_{n=1}^{\infty} \gamma_{n}<\infty \\
& \sum_{n=1}^{\infty} \beta_{n}=\infty, \sum_{n=1}^{\infty} \beta_{n}^{2}<\infty
\end{aligned}
$$

and assumptions H1a, H1b, H2 with $\sum_{n=1}^{\infty} \frac{a_{n}}{\sqrt{n}}<\infty$ replaced by $\sum_{n=1}^{\infty} a_{n} \sqrt{\beta_{n}}<\infty$. Condition (18) is verified for example when $\beta_{n}=\frac{1}{n}$ or from a certain rank when $\beta_{n}=\frac{1}{n^{\alpha}}, \frac{1}{2}<\alpha<1$. Note that the same time-scale can be taken for the two processes $\left(X_{n}\right)$ and $\left(M_{n}\right), \beta_{n}=a_{n}$ verifying $\sum_{n=1}^{\infty}\left(a_{n}\right)^{\frac{3}{2}}<\infty$.

In conclusion, solving the minimization of $F(x)$ in equations (6) and (8) involves the composition of a stochastic gradient descent algorithm and an iterative weighted algorithm, although the performed convergence analyses are different, allowing a larger choice of step-sizes in the present study without assumption on the comparison of the speed of convergence of $\left(a_{n}\right)$ and $\left(\beta_{n}\right)$. For example, for $a_{n}=1 / n^{\alpha}$ and $\beta_{n}=1 / n^{\beta}$, we must have: (i) in the case of SCGD, to verify assumption (15): $(1 / 2<\alpha \leq 1,1 / 2<$ 
$\beta \leq 1,2 \alpha-\beta>1) \Leftrightarrow(3 / 4<\alpha \leq 1,1 / 2<\beta<2 \alpha-1 \leq \alpha)$; (ii) in the case of our algorithm, to verify assumptions $\mathrm{H} 2$ and (18): $(1 / 2<\alpha \leq 1,1 / 2<\beta \leq 1, \alpha+\beta / 2>1)$, which is less restrictive; for $\beta=1,1 / 2<\alpha \leq 1$.

E. Note that Theorem 2.1 can be extended to the case where the explanatory variables have an expectation and a variance depending on $n$ or on the values of controlled variables, thus $m$ and $\Gamma$ depend on $n$ and are denoted by $m(n)$ and $\Gamma(n)$. Then $\hat{R}_{n-1}$ and $\hat{\Gamma}_{n-1}$ must be replaced by estimators of $m(n)$ and $\Gamma(n)$, respectively $\Theta_{n}$ and $\Phi_{n}$ depending on data up to step $n-1$ (as in [16] in the case of a time-varying expectation). To ensure the a.s. convergence of the process $\left(X_{n}\right)$, we assume that:

$$
\begin{aligned}
& \sup _{n}\|m(n)\|<\infty, \sup _{n}\|\Gamma(n)\|<\infty, \\
& \Theta_{n}-m(n) \longrightarrow 0, \sum_{n=1}^{\infty} a_{n}\left\|\Theta_{n}-m(n)\right\|<\infty, \\
& \Gamma_{n}-\Gamma(n) \longrightarrow 0, \sum_{n=1}^{\infty} a_{n}\left\|\Gamma_{n}-\Gamma(n)\right\|<\infty \text { a.s. }
\end{aligned}
$$

\section{Application}

Twenty-four stochastic approximation processes were compared, including classical stochastic gradient descent (SGD), averaged stochastic gradient descent (ASGD) with a piecewise constant step-size with different level sizes as suggested in [1], as well as the same processes but with the online standardization of the data defined in Subsection 2.1 .

The processes and their respective parameters are described in Table 1. Abbreviations used to name the processes are as follows: C for classical SGD or A for ASGD; $\mathrm{R}$ for raw data or $\mathrm{S}$ for online standardized data; $\mathrm{V}$ for variable step-size or $\mathrm{P}$ for piecewise constant step-size. For instance, AR1P50 is the averaged process with raw data, 1 observation per step, piecewise constant step-size with level size 50; CS1V is the classical process with online standardized data, 1 observation per step and variable step-size. Processes on raw data (in particular "CR.") are those currently used, while those using online standardization of the data (particularly averaged stochastic gradient descent with piecewise constant step-size, "AS.P."), are introduced in this article.

\subsection{Step-size}

For processes with a variable step-size $(\mathrm{V})$, we have defined $a_{n}=\frac{c}{(b+n)^{\alpha}}$. For processes with a piecewise constant step-size $(\mathrm{P})$, we have chosen $a_{n}=\frac{c}{\left(b+\left\lfloor\frac{n}{\tau}\right\rfloor\right)^{\alpha}}$ where $\lfloor$.$\rfloor denotes$ the integer part while $\tau$ is the size of the levels. For both cases, we set $\alpha=2 / 3$ (as suggested by $\mathrm{Xu}[24]$ in the case of linear regression), $b=1$ and $c=1$.

\subsection{Initialization and simulation of a data stream}

All processes were initialized with $X_{1}=0$. For processes with online standardization, a random sample of 1000 observations (drawn with replacement from the dataset) was used to compute a first estimation of the means and standard deviations of the 
Table 1. Description of the processes.

\begin{tabular}{|c|c|c|c|c|c|c|}
\hline Abbreviation & Method type & Type of data & $\begin{array}{l}\text { Number of } \\
\text { observations } \\
\text { used at each } \\
\text { step of the } \\
\text { process }\end{array}$ & Step-size & $\begin{array}{l}\text { Levels } \\
\text { size }\end{array}$ & $\begin{array}{l}\text { Use of the } \\
\text { averaged } \\
\text { process }\end{array}$ \\
\hline CR1V & \multirow{3}{*}{ Classical (C) } & \multirow{12}{*}{ Raw data (R) } & 1 & \multirow{3}{*}{ Variable $(\mathrm{V})$} & \multirow{3}{*}{-} & \multirow{3}{*}{ No } \\
\hline CR10V & & & 10 & & & \\
\hline CR100V & & & 100 & & & \\
\hline AR1P50 & \multirow{9}{*}{$\boldsymbol{A}$ veraged (A) $S G D$} & & 1 & \multirow{9}{*}{$\begin{array}{l}\text { Piecewise } \\
\text { constant }(\mathrm{P})\end{array}$} & \multirow{3}{*}{50} & \multirow{9}{*}{ Yes } \\
\hline AR10P50 & & & 10 & & & \\
\hline AR100P50 & & & 100 & & & \\
\hline AR1P100 & & & 1 & & \multirow{3}{*}{100} & \\
\hline AR10P100 & & & 10 & & & \\
\hline AR100P100 & & & 100 & & & \\
\hline AR1P200 & & & 1 & & \multirow{3}{*}{200} & \\
\hline AR10P200 & & & 10 & & & \\
\hline AR100P200 & & & 100 & & & \\
\hline CS1V & \multirow{3}{*}{ Classical (C) } & \multirow{12}{*}{$\begin{array}{c}\text { Online } \\
\text { Standardized } \\
\text { data }(\mathrm{S})\end{array}$} & 1 & \multirow{3}{*}{ Variable (V) } & \multirow{3}{*}{-} & \multirow{3}{*}{ No } \\
\hline CS10V & & & 10 & & & \\
\hline CS100V & & & 100 & & & \\
\hline AS1P50 & \multirow{9}{*}{$\boldsymbol{A}$ veraged (A) $S G D$} & & 1 & \multirow{9}{*}{$\begin{array}{l}\text { Piecewise } \\
\text { constant }(\mathrm{P})\end{array}$} & \multirow{3}{*}{50} & \multirow{9}{*}{ Yes } \\
\hline AS10P50 & & & 10 & & & \\
\hline AS100P50 & & & 100 & & & \\
\hline AS1P100 & & & 1 & & & \\
\hline AS10P100 & & & 10 & & 100 & \\
\hline AS100P100 & & & 100 & & & \\
\hline AS1P200 & & & 1 & & & \\
\hline AS10P200 & & & 10 & & 200 & \\
\hline AS100P200 & & & 100 & & & \\
\hline
\end{tabular}

explanatory variables prior to the beginning of the iterations. For averaged processes, the first 1000 iterations were used as a burn-in period and were not included in the computation of the average.

Then, for each dataset, a data stream was simulated by randomly sampling with replacement a data batch of 1, 10 or 100 observations (depending on the process studied) at each step of the process.

Processes were implemented with the R 3.6.1 software (64-bit version).

\subsection{Convergence criteria}

The coefficients obtained by the usual "offline" logistic regression (using R's glm function) on a dataset $\left(\left(r_{i}^{1}, \ldots, r_{i}^{p}, s_{i}\right), i=1, \ldots, N\right)$ were used as "gold standard" to assess the convergence of the processes. Let $\theta^{c}$ be the vector of coefficients obtained with this method and $\hat{\theta}_{n+1}$ the estimated vector obtained by a tested process after $n$ iterations.

$\operatorname{As} \theta_{0}=\left(\begin{array}{cccc}\frac{1}{\sigma^{1}} & & \\ & \ddots & & \\ & & \frac{1}{\sigma^{p}} & \\ -\frac{m^{1}}{\sigma^{1}} & \cdots & -\frac{m^{p}}{\sigma^{p}} & 1\end{array}\right) \theta, \hat{\theta}_{n+1}=\left(\begin{array}{cccc}\hat{\Gamma}_{n}(1,1) & & \\ & \ddots & & \\ & & \hat{\Gamma}_{n}(p, p) & \\ -\hat{\Gamma}_{n}(1,1) \hat{r}_{n}^{1} & \cdots & -\hat{\Gamma}_{n}(p, p) \hat{r}_{n}^{p} & 1\end{array}\right) \bar{x}_{n+1}$

$\left(\bar{x}_{n+1}\right.$, realization of $\bar{X}_{n+1}$, is the estimation of $\theta$ at step $\left.\mathrm{n}\right)$.

The relative norm of the difference between $\theta^{c}$ and $\hat{\theta}_{n+1}, \frac{\left\|\theta^{c}-\hat{\theta}_{n+1}\right\|}{\left\|\theta^{c}\right\|}$, was used as a convergence criterion. 
The cosine of the angle between $\theta^{c}$ and $\hat{\theta}_{n+1}, \frac{\theta^{c} \hat{\theta}_{n+1}}{\left\|\theta^{c}\right\|\left\|\hat{\theta}_{n+1}\right\|}$, the coefficient of correlation between the predictions obtained with the usual method and the process, as well as the ratio $\frac{\hat{F}\left(\hat{\theta}_{n+1}\right)-\hat{F}\left(\theta^{c}\right)}{\hat{F}\left(\theta^{c}\right)}, \hat{F}\left(\hat{\theta}_{n+1}\right)=\frac{1}{N} \sum_{i=1}^{N}\left(-r_{i}^{\prime} \hat{\theta}_{n+1} s_{i}+\ln \left(1+e^{r_{i} \hat{\theta}_{n+1}}\right)\right)$ being an estimation of the cost function $F$ at $\hat{\theta}_{n+1}$, were also used as criteria (results not shown).

\subsection{Datasets}

The processes were tested on four datasets available on the Internet and one dataset derived from the EPHESUS study $[20]^{1}$, all of which have already been used to test the performance of stochastic approximation processes with online standardized data in the case of online linear regression [10]. Twonorm, Ringnorm, Quantum and Adult datasets are commonly used to test classification methods. Twonorm ${ }^{2}$ and Ringnorm ${ }^{3}$, introduced by Breiman [5], contain simulated data with homogeneous variables. Quantum contains observed "clean" data, without outliers and with most of its variables on a similar scale. Adult and HOSPHF30D contain observed data with outliers, heterogeneous variables of different types and scales. Table 2 summarizes these datasets.

Table 2. Description of the datasets.

\begin{tabular}{lccccl}
\hline Dataset name & $N_{a}$ & $N$ & $p_{a}$ & $p$ & Source \\
\hline Twonorm & 7400 & 7400 & 20 & 20 & www.cs.toronto.edu/ delve/data/datasets.html \\
\hline Ringnorm & 7400 & 7400 & 20 & 20 & www.cs.toronto.edu/ delve/data/datasets.html \\
\hline Quantum & 50000 & 15798 & 78 & 12 & derived from www.osmot.cs.cornell.edu/kddcup \\
\hline Adult2 & 45222 & 45222 & 14 & 38 & derived from www.cs.toronto.edu/delve/data/datasets.html \\
\hline HOSPHF30D & 21382 & 21382 & 29 & 13 & derived from EPHESUS study [20] \\
\hline
\end{tabular}

$N_{a}$ : number of available observations; $N$ : number of selected observations; $p_{a}$ : number of available parameters; $p$ : number of selected parameters.

The following preprocessings were performed on the data:

- Twonorm and Ringnorm: no preprocessing.

- Quantum: a stepwise variable selection (using AIC) was performed on the 6197 observations without any missing value. The dataset with complete observations for the 12 selected variables was used.

- Adult2: from the Adult dataset, modalities of several categorical variables were merged (in order to obtain a larger number of observations for each modality) and all categorical variables were then replaced by sets of binary variables, leading to a dataset with 38 variables.

- HOSPHF30D: 13 variables were selected using stepwise selection.

All processes were applied on all datasets for a fixed number of $100 \mathrm{~N}$ observations used and for a fixed processing time of 60s (the cumulative time to compute the process updates, excluding operations such as data sampling, data management, formatting and recording of results, etc.).

\footnotetext{
${ }^{1}$ Due to legal restrictions, data from the EPHESUS study are only available upon request. Interested researchers may request access to data upon approval from the EPHESUS Executive Steering Committee of the study.

${ }^{2}$ Twonorm "is 20 dimension, 2 class data. Each class is drawn from a multivariate normal distribution with unit covariance matrix. Class 1 has mean $(a, a, \ldots, a)$ and class 2 has mean $(-a,-a, \ldots,-a) . "($ extract from [5])

${ }^{3}$ Ringnorm "is 20 dimension, 2 class data. Class 1 is multivariate normal with mean zero and covariance matrix 4 times the identity. Class 2 has unit covariance matrix and mean $(a, a, \ldots, a)$."(extract from [5])
} 
For each dataset and at each recording point (see below), processes that did not explode were ranked from the best (lowest relative norm) to the worst (highest relative norm). The mean rank over all datasets was used to compare the global performance of the processes without any numerical explosion.

Processing time to treat $100 \mathrm{~N}$ observations and average number of observations used per second were also studied. Note that it is preferable to consider only the order of magnitude of these indicators, since CPU and memory usage by other software were not controlled during the running of the processes which may explain small differences.

\subsection{Comparison for a fixed number of observations}

As in [10], the criteria values for each process were recorded every $N$ observations used, from $1 N$ to $100 N, N$ being the number of selected observations after preprocessing in the studied dataset. For the relative norm criterion, results for $100 \mathrm{~N}$ observations are shown in Table 3. Note that since the number of observations used at each step differs from one process to another, the number of iterations is not the same for each process (e.g. to use $100 N$ observations, CR1V will run for $100 N$ iterations whereas CR100V will run for $\mathrm{N}$ iterations).

Table 3. Relative norms for $100 N$ observations used

\begin{tabular}{|c|c|c|c|c|c|c|}
\hline Process & Twonorm & Ringnorm & Quantum & Adult & HOSPHF30D & Mean rank \\
\hline CR1V & 0.085 & $0.026^{*}$ & 0.304 & EXPL & EXPL & - \\
\hline CR10V & 0.206 & $0.017^{*}$ & 0.500 & EXPL & EXPL & - \\
\hline CR100V & 0.335 & $0.019^{*}$ & 0.661 & EXPL & EXPL & - \\
\hline AR1P50 & $0.028^{*}$ & $0.037^{*}$ & 0.087 & EXPL & EXPL & - \\
\hline AR10P50 & $0.010^{*}$ & $0.013^{*}$ & 0.118 & EXPL & EXPL & - \\
\hline AR100P50 & $0.034^{*}$ & $0.006^{*}$ & 0.191 & EXPL & EXPL & - \\
\hline AR1P100 & $0.035^{*}$ & 0.061 & 0.065 & EXPL & EXPL & - \\
\hline AR10P100 & $0.010^{*}$ & $0.021^{*}$ & 0.102 & EXPL & EXPL & - \\
\hline AR100P100 & $0.014^{*}$ & $0.007^{*}$ & 0.144 & EXPL & EXPL & - \\
\hline AR1P200 & $0.040^{*}$ & 0.102 & $0.041^{*}$ & EXPL & EXPL & - \\
\hline AR10P200 & $0.012^{*}$ & $0.034^{*}$ & 0.090 & EXPL & EXPL & - \\
\hline AR100P200 & $0.009^{*}$ & $0.011^{*}$ & 0.123 & EXPL & EXPL & - \\
\hline CS1V & 0.108 & $0.016^{*}$ & 0.074 & 0.057 & 0.088 & 10.2 \\
\hline CS10V & 0.234 & $0.011^{*}$ & $0.041^{*}$ & 0.085 & 0.260 & 9.8 \\
\hline CS100V & 0.364 & $0.009^{*}$ & 0.138 & 0.120 & 0.629 & 10.6 \\
\hline AS1P50 & $0.016^{*}$ & $0.015^{*}$ & $0.027^{*}$ & $0.035^{*}$ & 0.064 & 6.6 \\
\hline AS10P50 & $0.011^{*}$ & $0.008^{*}$ & $0.024^{*}$ & $0.011^{*}$ & 0.060 & 3.2 \\
\hline AS100P50 & $0.048^{*}$ & $0.006^{*}$ & $0.024^{*}$ & $0.021^{*}$ & 0.067 & 5.8 \\
\hline AS1P100 & $0.018^{*}$ & $0.023^{*}$ & $0.035^{*}$ & $0.040^{*}$ & 0.062 & 7.6 \\
\hline AS10P100 & $0.010^{*}$ & $0.010^{*}$ & $0.024^{*}$ & $0.014^{*}$ & 0.060 & 3.8 \\
\hline AS100P100 & $0.021^{*}$ & $0.006^{*}$ & $0.023^{*}$ & $0.013^{*}$ & 0.065 & 4.2 \\
\hline AS1P200 & $0.026^{*}$ & $0.036^{*}$ & $0.049^{*}$ & 0.055 & 0.057 & 8.0 \\
\hline AS10P200 & $0.011^{*}$ & $0.015^{*}$ & $0.028^{*}$ & $0.018^{*}$ & 0.059 & 5.2 \\
\hline AS100P200 & $0.010^{*}$ & $0.007^{*}$ & $0.022^{*}$ & $0.011^{*}$ & 0.066 & 3.0 \\
\hline
\end{tabular}

* denotes a criterion value $<0.05$.

EXPL: numerical explosion.

Process type: C for classical SGD, A for ASGD. Data type: R for raw data, S for online standardized data. First number: number of new observations at each step.

Step-size: V for variable, $\mathrm{P}$ for piecewise constant (second number denotes the level size).

Globally, processes $\mathrm{R}$ and $\mathrm{S}$ converged similarly on simulated datasets with homogeneous data (especially Twonorm which already contains standardized variables). However, it was not verified for observed datasets for which processes S yielded better results, especially with heterogeneous data (Adult2, HOSPHF30D): all tested processes $\mathrm{R}$ had a numerical explosion for these two datasets.

Over all datasets, the processes $\mathrm{S}$ with the lowest mean rankings were averaged 
processes with piecewise constant step-sizes, the best being AS100P200 (Table 4). Among these AS.P. processes, those with the highest mean rankings were processes with one observation per step (AS1P. ). Note that for HOSPHF30D, all AS.P. processes had a criterion value lower than 0.05 after $300 N$ observations used (Appendix Table B1).

Table 4. Processes $\mathrm{S}$ ordered by mean ranks for $100 N$ observations used

\begin{tabular}{lcccccc}
\hline Process & AS100P200 & AS10P50 & AS10P100 & AS100P100 & AS10P200 & AS100P50 \\
Mean rank & 3.0 & 3.2 & 3.8 & 4.2 & 5.2 & 5.8 \\
\hline Process & AS1P50 & AS1P100 & AS1P200 & CS10V & CS1V & CS100V \\
Mean rank & 6.6 & 7.6 & 8.0 & 9.8 & 10.2 & 10.6 \\
\hline
\end{tabular}

Additional results for both fixed and varying numbers of observations are available as Supplemental online material.

\subsection{Comparison for a fixed processing time}

As in [10], the values of the criteria for each process were then recorded every second of processing time from 1 to 120s. Results for the relative norm criterion after 60s of processing time are shown in Table 5.

Table 5. Relative norms for 60 s of processing time

\begin{tabular}{|c|c|c|c|c|c|c|}
\hline Process & Twonorm & Ringnorm & Quantum & Adult & HOSPHF30D & Mean rank \\
\hline CR1V & 0.055 & $0.019^{*}$ & 0.288 & EXPL & EXPL & - \\
\hline CR10V & 0.061 & $0.005^{*}$ & 0.310 & EXPL & EXPL & - \\
\hline CR100V & 0.073 & $0.002^{*}$ & 0.333 & EXPL & EXPL & - \\
\hline AR1P50 & $0.011^{*}$ & $0.019^{*}$ & 0.086 & EXPL & EXPL & - \\
\hline AR10P50 & $0.002^{*}$ & $0.002^{*}$ & 0.095 & EXPL & EXPL & - \\
\hline AR100P50 & $0.001^{*}$ & $0.001^{*}$ & 0.102 & EXPL & EXPL & - \\
\hline AR1P100 & $0.015^{*}$ & $0.029^{*}$ & 0.064 & EXPL & EXPL & - \\
\hline AR10P100 & $0.002^{*}$ & $0.003^{*}$ & 0.079 & EXPL & EXPL & - \\
\hline AR100P100 & $0.001^{*}$ & $0.001^{*}$ & 0.090 & EXPL & EXPL & - \\
\hline AR1P200 & $0.018^{*}$ & 0.052 & $0.040^{*}$ & EXPL & EXPL & - \\
\hline AR10P200 & $0.002^{*}$ & $0.005^{*}$ & 0.064 & EXPL & EXPL & - \\
\hline AR100P200 & $0.001^{*}$ & $0.001^{*}$ & 0.076 & EXPL & EXPL & - \\
\hline CS1V & 0.139 & $0.023^{*}$ & 0.173 & 0.134 & 0.153 & 10.0 \\
\hline CS10V & 0.182 & $0.011^{*}$ & 0.057 & 0.101 & 0.228 & 9.0 \\
\hline CS100V & 0.227 & $0.004^{*}$ & 0.071 & 0.108 & 0.326 & 9.0 \\
\hline AS1P50 & $0.027^{*}$ & $0.025^{*}$ & $0.042^{*}$ & 0.389 & 0.095 & 8.6 \\
\hline AS10P50 & $0.006^{*}$ & $0.005^{*}$ & $0.014^{*}$ & $0.020^{*}$ & 0.053 & 4.8 \\
\hline AS100P50 & $0.009^{*}$ & $0.002^{*}$ & $0.007^{*}$ & $0.017^{*}$ & $0.014^{*}$ & 3.2 \\
\hline AS1P100 & $0.032^{*}$ & $0.037^{*}$ & 0.071 & 0.386 & 0.087 & 9.2 \\
\hline AS10P100 & $0.005^{*}$ & $0.006^{*}$ & $0.014^{*}$ & $0.025^{*}$ & $0.050^{*}$ & 4.8 \\
\hline AS100P100 & $0.004^{*}$ & $0.002^{*}$ & $0.007^{*}$ & $0.011^{*}$ & $0.011^{*}$ & 1.8 \\
\hline AS1P200 & $0.046^{*}$ & 0.060 & 0.121 & 0.498 & 0.112 & 10.6 \\
\hline AS10P200 & $0.005^{*}$ & $0.008^{*}$ & $0.017^{*}$ & $0.035^{*}$ & $0.049^{*}$ & 5.4 \\
\hline AS100P200 & $0.003^{*}$ & $0.002^{*}$ & $0.007^{*}$ & $0.009^{*}$ & $0.012^{*}$ & 1.6 \\
\hline
\end{tabular}

* denotes a criterion value $<0.05$.

EXPL: numerical explosion.

Process type: C for classical SGD, A for ASGD. Data type: R for raw data, S for online standardized data. First number: number of new observations at each step.

Step-size: $\mathrm{V}$ for variable, $\mathrm{P}$ for piecewise constant (second number denotes the level size).

Again, all tested processes $\mathrm{R}$ had a numerical explosion for the same two datasets (Adult2 and HOSHF30D).

Over all datasets, processes $\mathrm{S}$ with the lowest mean rankings were averaged processes with piecewise constant step-sizes (Table 6), except AS1P100 and AS1P200 with one 
observation per step (which use few observations per second, see Table 7). The best process was AS100P200. Of note, for HOSPHF30D, processes AS10P. and AS100P. had a criterion value lower than 0.05 at 120s (Appendix Table B2).

Table 6. Processes $\mathrm{S}$ ordered by mean ranks for 60 s of processing time

\begin{tabular}{lcccccc}
\hline Process & AS100P200 & AS100P100 & AS100P50 & AS10P50 & AS10P100 & AS10P200 \\
Mean rank & 1.6 & 1.8 & 3.2 & 4.8 & 4.8 & 5.4 \\
\hline Process & AS1P50 & CS10V & CS100V & AS1P100 & CS1V & AS1P200 \\
Mean rank & 8.6 & 9.0 & 9.0 & 9.2 & 10.0 & 10.6 \\
\hline
\end{tabular}

The average numbers of observations used per second for 60s of processing time are shown in Table 7. For all processes, the number of observations used per second increased with the number of observations used at each step. Due to the online updating of expectations and variances, processes $S$ treated less observations per second than their equivalent on raw data, the ratio $n_{R} / n_{S}$ increasing with the number of observations used at each step $\left(n_{R}\right.$, resp. $n_{S}$, the number of observations treated per second by a process $\mathrm{R}$, resp. S).

These results combined with those of Table 5 indicate that processes $\mathrm{S}$ with piecewise constant step-sizes, particularly AS100P. and AS10P., achieved a better performance using less observations.

Table 7. Average number of observations used per second for 60 s of processing time

\begin{tabular}{lccccc}
\hline Process & Twonorm & Ringnorm & Quantum & Adult & HOSPHF30D \\
\hline CR1V & 32464 & 31661 & 32632 & EXPL & EXPL \\
\hline CR10V & 307385 & 291475 & 292206 & EXPL & EXPL \\
\hline CR100V & 2189952 & 2016338 & 2248173 & EXPL & EXPL \\
\hline AR1P50 & 32019 & 29106 & 30247 & EXPL & EXPL \\
\hline AR10P50 & 273377 & 303805 & 283962 & EXPL & EXPL \\
\hline AR100P50 & 2169318 & 2226950 & 2189662 & EXPL & EXPL \\
\hline AR1P100 & 31433 & 33362 & 30591 & EXPL & EXPL \\
\hline AR10P100 & 282423 & 292126 & 283747 & EXPL & EXPL \\
\hline AR100P100 & 2127127 & 2248095 & 2210533 & EXPL & EXPL \\
\hline AR1P200 & 29100 & 30919 & 29420 & EXPL & EXPL \\
\hline AR10P200 & 271102 & 275657 & 273606 & EXPL & EXPL \\
\hline AR100P200 & 2077237 & 2019090 & 2129145 & EXPL & EXPL \\
\hline CS1V & 5970 & 6061 & 6162 & 5799 & 6715 \\
\hline CS10V & 33469 & 34216 & 35776 & 29430 & 41679 \\
\hline CS100V & 142573 & 141815 & 154233 & 119468 & 169937 \\
\hline AS1P50 & 5862 & 5856 & 5635 & 5613 & 6560 \\
\hline AS10P50 & 33780 & 34336 & 35548 & 31498 & 43315 \\
\hline AS100P50 & 139907 & 142993 & 154767 & 119863 & 190613 \\
\hline AS1P100 & 6030 & 6031 & 5994 & 5819 & 7059 \\
\hline AS10P100 & 33884 & 34075 & 34904 & 32019 & 42846 \\
\hline AS100P100 & 141037 & 144380 & 151340 & 121745 & 180893 \\
\hline AS1P200 & 5819 & 5871 & 5978 & 5606 & 7016 \\
\hline AS10P200 & 34032 & 34474 & 34638 & 31910 & 44620 \\
\hline AS100P200 & 133773 & 140265 & 149313 & 108710 & 193833 \\
\hline
\end{tabular}

EXPL: numerical explosion.

Process type: C for classical SGD, A for ASGD. Data type: R for raw data, S for online standardized data.

First number: number of new observations at each step.

Step-size: $\mathrm{V}$ for variable, $\mathrm{P}$ for piecewise constant (second number denotes the level size). 


\subsection{Comparison of processes $S$ for a varying processing time}

When studying the evolution of the mean rankings of processes $\mathrm{S}$ with processing times from 1 to 120s (Figure 1), two groups of processes clearly appeared from the outset and remained apparent throughout the entire studied period. The group with the worst rankings contains all processes using only one new observation at each step as well as all "classical" processes. The group with the best rankings contained all averaged processes S using 10 or 100 new observations at each step. Within this group, a clear difference appeared after approximately 10s of processing time between processes using 10 new observations and those using 100 new observations. Of all the processing times recorded, the two best processes S appeared to be AS100P100 and AS100P200.

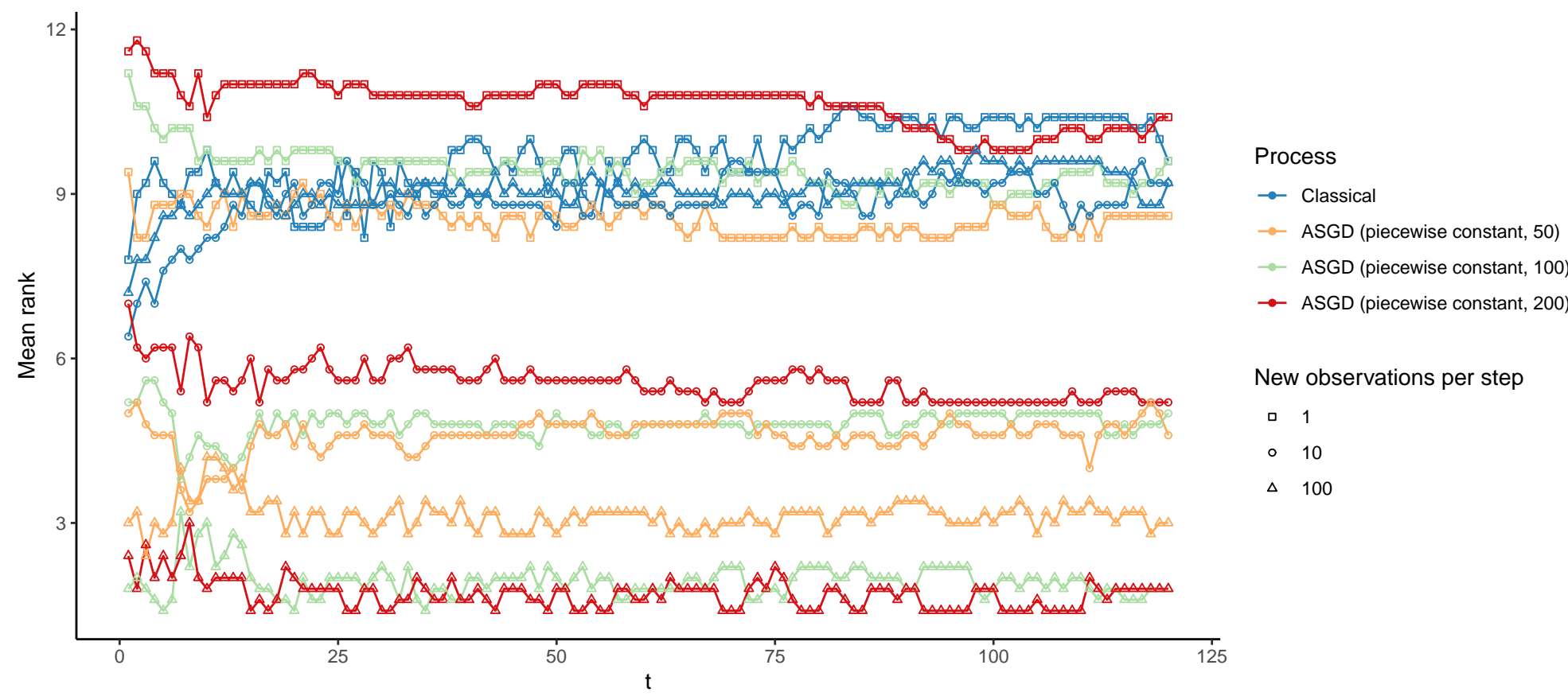

Figure 1. Evolution according to processing time

\section{Conclusion}

In the present analysis, we studied a constrained averaged stochastic gradient algorithm with online standardized data for performing an online constrained binary logistic regression in the case of streaming or massive data. The proposed approach included using an online standardization of the data to avoid a numerical explosion, or when using a shrinkage method (such as LASSO), or even when expectations or variances of explanatory variables change (varying with time or depending on the values of controlled variables) and can be estimated online. We also proposed using a decreasing piecewise constant step-size in order for the latter to not decrease too quickly and therefore not reduce the speed of convergence of the process. The results of the experiments conducted on real and simulated datasets confirm the validity of the choices made: online standardization of the data, averaged process and piecewise constant step-size. This work will be applied in an ongoing study to update an ensemble 
score online to detect adverse events in the case of heart failure $[8,9]$.

\section{Acknowledgments}

The authors thank Mr. Pierre Pothier and Mr. Edward Sismey for editing this manuscript.

\section{Disclosure statement}

No potential conflict of interest was reported by the authors.

\section{Funding}

This work was supported by the investments for the Future Program under grant ANR-15-RHU-0004.

\section{References}

[1] F. Bach, Adaptivity of averaged stochastic gradient descent to local strong convexity for logistic regression, Journal of Machine Learning Research 15 (2014), pp. 595-627.

[2] F. Bach and E. Moulines, Non-strongly-convex smooth stochastic approximation with convergence rate $O(1 / n)$, in Advances in Neural Information Processing Systems 26, C.J.C. Burges, L. Bottou, M. Welling, Z. Ghahramani, and K.Q. Weinberger, eds., Curran Associates, Inc., 2013, pp. 773-781.

[3] L. Bottou, F. Curtis, and J. Nocedal, Optimization methods for large-scale machine learning, SIAM Review 60 (2018), pp. 223-311.

[4] L. Bottou and Y. Le Cun, On-line learning for very large data sets, Applied stochastic models in business and industry 21 (2005), pp. 137-151.

[5] L. Breiman, Bias, variance, and arcing classifiers, Technical Report 460, Departement of Statistics, University of California, Berkeley (1996).

[6] H. Cardot, P. Cénac, and J.M. Monnez, A fast and recursive algorithm for clustering large datasets with $k$-medians, Computational Statistics \& Data Analysis 56 (2012), pp. 1434-1449.

[7] D. Dentcheva, S. Penev, and A. Ruszczyński, Statistical estimation of composite risk functionals and risk optimization problems, Annals of the Institute of Statistical Mathematics 69 (2017), pp. 737-760.

[8] K. Duarte, Medical decision support and telemedecine in the monitoring of heart failure. Ph.D. thesis., Université de Lorraine (France) (2018). Available at https://hal.univlorraine.fr/tel-02096008.

[9] K. Duarte, J.M. Monnez, and E. Albuisson, Methodology for constructing a short-term event risk score in heart failure patients, Applied Mathematics 09 (2018), pp. 954-974.

[10] K. Duarte, J.M. Monnez, and E. Albuisson, Sequential linear regression with online standardized data, PLOS ONE 13 (2018), p. e0191186.

[11] S. Ghadimi, A. Ruszczyński, and M. Wang, A Single Time-Scale Stochastic Approximation Method for Nested Stochastic Optimization, arXiv:1812.01094 [math] (2019).

[12] Y. Hu, X. Chen, and N. He, Sample Complexity of Sample Average Approximation for Conditional Stochastic Optimization, arXiv:1905.11957 [math, stat] (2020).

[13] R. Johnson and T. Zhang, Accelerating stochastic gradient descent using predictive variance reduction, in Advances in Neural Information Processing Systems 26, C.J.C. Burges, 
L. Bottou, M. Welling, Z. Ghahramani, and K.Q. Weinberger, eds., Curran Associates, Inc., 2013, pp. 315-323.

[14] L. Ljung, G.C. Pflug, and H. Walk, Stochastic approximation and optimization of random systems, DMV Seminar, Band 17, Birkhäuser, Basel, 1992.

[15] J.M. Monnez, Approximation stochastique en analyse factorielle multiple (Stochastic approximation in multiple factor analysis), Annales de l'ISUP 50 (2006), pp. 27-45.

[16] J.M. Monnez, Analyse en composantes principales d'un flux de données d'espérance variable dans le temps (Principal component analysis of a data stream with time-varying expectation), Revue des Nouvelles Technologies de l'Information (2008), pp. 43-56.

[17] J.M. Monnez, Stochastic approximation of the factors of a generalized canonical correlation analysis, Statistics and Probability Letters 78 (2008), pp. 2210-2216.

[18] J.M. Monnez and A. Skiredj, Convergence of a normed eigenvector stochastic approximation process and application to online principal component analysis of a data stream, 2018. Available at https://hal.archives-ouvertes.fr/hal-01844419.

[19] R. Pascanu, T. Mikolov, and Y. Bengio, Understanding the exploding gradient problem, arXiv:1211.5063 (2012). Available at http://arxiv.org/abs/1211.5063.

[20] B. Pitt, W. Remme, F. Zannad, J. Neaton, F. Martinez, B. Roniker, R. Bittman, S. Hurley, J. Kleiman, and M. Gatlin, Eplerenone, a selective aldosterone blocker, in patients with left ventricular dysfunction after myocardial infarction, New England Journal of Medicine 348 (2003), pp. 1309-1321.

[21] B.T. Polyak and A.B. Juditsky, Acceleration of stochastic approximation by averaging, SIAM Journal on Control and Optimization 30 (1992), pp. 838-855.

[22] H. Robbins and D. Siegmund, A convergence theorem for nonnegative almost supermartingales and some applications, in Optimizing Methods in Statistics, J.S. Rustagi, ed., Academic Press, 1971, pp. 233-257.

[23] M. Wang, E.X. Fang, and H. Liu, Stochastic compositional gradient descent: algorithms for minimizing compositions of expected-value functions, Mathematical Programming 161 (2017), pp. 419-449.

[24] W. Xu, Towards optimal one pass large scale learning with averaged stochastic gradient descent, arXiv:1107.2490 (2011). Available at http://arxiv.org/abs/1107.2490.

[25] S. Yang, M. Wang, and X.E. Fang, Multilevel stochastic gradient methods for nested composition optimization, SIAM Journal on Optimization 29 (2019), pp. 616-659.

\section{Appendix A. Proof of the convergence theorem}

The usual Euclidean norm in $\mathbb{R}^{p+1}$ and the spectral norm for matrices are used in this proof. Let us state the Robbins-Siegmund lemma [22] and another lemma ([10], Lemma 5) used in this proof. This proof is also valid in the case of linear regression.

Lemma A.1. Let $(\Omega, A, P)$ be a probability space and $\left(T_{n}\right)$ a non-decreasing sequence of sub- $\sigma$-fields of $A$. Suppose for all $n, z_{n}, \alpha_{n}, \beta_{n}$ and $\gamma_{n}$ are four integrable nonnegative $T_{n}$-measurable random variables defined on $(\Omega, A, P)$ such that:

$$
E\left[z_{n+1} \mid T_{n}\right] \leq z_{n}\left(1+\alpha_{n}\right)+\beta_{n}-\gamma_{n} \text { a.s. }
$$

Then, in the set $\left\{\sum_{n=1}^{\infty} \alpha_{n}<\infty, \sum_{n=1}^{\infty} \beta_{n}<\infty\right\},\left(z_{n}\right)$ converges a.s. to a finite random variable and $\sum_{n=1}^{\infty} \gamma_{n}<\infty$ a.s. 
Lemma A.2. Suppose H1b holds and $a_{n}>0, \sum_{n=1}^{\infty} \frac{a_{n}}{\sqrt{n}}<\infty$. Then:

$$
\sum_{n=1}^{\infty} a_{n}\left\|\widehat{R}_{n-1}^{c}\right\|<\infty \text { and } \sum_{n=1}^{\infty} a_{n}\left\|\widehat{\Gamma}_{n-1}-\Gamma\right\|<\infty \text { a.s. }
$$

Proof. Part 1. Let $T_{n}$ be the $\sigma$-field generated by the events before time $n: X_{1}, \ldots, X_{n}$ are $T_{n}$-measurable, as $\widehat{R}_{n-1}$ and $\widehat{\Gamma}_{n-1}$.

$$
\begin{aligned}
\left\|X_{n+1}-\theta\right\| & =\left\|\Pi\left(X_{n}-a_{n} \frac{1}{m_{n}} \sum_{j \in I_{n}} \widetilde{Z}_{j}\left(h\left(\widetilde{Z}_{j}^{\prime} X_{n}\right)-S_{j}\right)\right)-\Pi \theta\right\| \\
& \leq\left\|X_{n}-a_{n} \frac{1}{m_{n}} \sum_{j \in I_{n}} \widetilde{Z}_{j}\left(h\left(\widetilde{Z}_{j}^{\prime} X_{n}\right)-S_{j}\right)-\theta\right\| .
\end{aligned}
$$

Taking the conditional expectation with respect to $T_{n}$ yields a.s.:

$$
\begin{aligned}
E\left[\left\|X_{n+1}-\theta\right\|^{2} \mid T_{n}\right] & \leq\left\|X_{n}-\theta\right\|^{2} \\
& -2 a_{n}\left\langle X_{n}-\theta, \frac{1}{m_{n}} \sum_{j \in I_{n}} E\left[\widetilde{Z}_{j}\left(h\left(\widetilde{Z}_{j}^{\prime} X_{n}\right)-S_{j}\right) \mid T_{n}\right]\right\rangle \\
& +a_{n}^{2} E\left[\left\|\frac{1}{m_{n}} \sum_{j \in I_{n}} \widetilde{Z}_{j}\left(h\left(\widetilde{Z}_{j}^{\prime} X_{n}\right)-S_{j}\right)\right\|^{2} \mid T_{n}\right] \text { a.s. }
\end{aligned}
$$

Part 2. Decomposition of $E\left[\widetilde{Z}_{j}\left(h\left(\widetilde{Z}_{j}^{\prime} X_{n}\right)-S_{j}\right) \mid T_{n}\right], j \in I_{n}$.

$$
\begin{aligned}
E\left[\widetilde{Z}_{j}\left(h\left(\widetilde{Z}_{j}^{\prime} X_{n}\right)-S_{j}\right) \mid T_{n}\right] & =E\left[\widetilde{Z}_{j}\left(h\left(\widetilde{Z}_{j}^{\prime} X_{n}\right)-E\left[S_{j} \mid Z_{j}\right]\right) \mid T_{n}\right]+E\left[\widetilde{Z}_{j}\left(S_{j}-E\left[S_{j} \mid Z_{j}\right]\right) \mid T_{n}\right] . \\
E\left[\widetilde{Z}_{j}\left(S_{j}-E\left[S_{j} \mid Z_{j}\right]\right) \mid T_{n}\right] & =E\left[\widehat{\Gamma}_{n-1}\left(R_{j}-\widehat{R}_{n-1}\right)\left(S_{j}-E\left[S_{j} \mid Z_{j}\right]\right) \mid T_{n}\right] \\
& =\widehat{\Gamma}_{n-1} E[R(S-E[S \mid Z])]-\widehat{\Gamma}_{n-1} \widehat{R}_{n-1} E[S-E[S \mid Z]] \\
& =0 \text { a.s. }
\end{aligned}
$$

Then: $E\left[\widetilde{Z}_{j}\left(h\left(\widetilde{Z}_{j}^{\prime} X_{n}\right)-S_{j}\right) \mid T_{n}\right]=E\left[\widetilde{Z}_{j}\left(h\left(\widetilde{Z}_{j}^{\prime} X_{n}\right)-h\left(Z_{j}^{\prime} \theta\right)\right) \mid T_{n}\right]$ a.s.

Consider the decomposition

$$
\begin{gathered}
E\left[\widetilde{Z}_{j}\left(h\left(\widetilde{Z}_{j}^{\prime} X_{n}\right)-h\left(Z_{j}^{\prime} \theta\right)\right) \mid T_{n}\right]=E\left[Z_{j}\left(h\left(Z_{j}^{\prime} X_{n}\right)-h\left(Z_{j}^{\prime} \theta\right)\right) \mid T_{n}\right]+E\left[V_{j} \mid T_{n}\right] \\
\quad \text { with } V_{j}=\left(\widetilde{Z}_{j}-Z_{j}\right)\left(h\left(Z_{j}^{\prime} X_{n}\right)-h\left(Z_{j}^{\prime} \theta\right)\right)+\widetilde{Z}_{j}\left(h\left(\widetilde{Z}_{j}^{\prime} X_{n}\right)-h\left(Z_{j}^{\prime} X_{n}\right)\right)
\end{gathered}
$$

For $j \in I_{n}$, there exist $0 \leq \lambda_{j} \leq 1, \xi_{j}^{1}$ and $\xi_{j}^{2}$ such that: 
a) $h\left(Z_{j}^{\prime} X_{n}\right)-h\left(Z_{j}^{\prime} \theta\right)=Z_{j}^{\prime}\left(X_{n}-\theta\right) h^{\prime}\left(\xi_{j}\right)$, with $\xi_{j}=\lambda_{j} Z_{j}^{\prime} X_{n}+\left(1-\lambda_{j}\right) Z_{j}^{\prime} \theta$, $Z_{j}=\Gamma R_{j}^{c}$;

b) $h\left(\widetilde{Z}_{j}^{\prime} X_{n}\right)=h\left(\left(R_{j}^{c}-\widehat{R}_{n-1}^{c}\right)^{\prime} \widehat{\Gamma}_{n-1} X_{n}\right)$

$$
\begin{aligned}
& =h\left(\left(R_{j}^{c}-\widehat{R}_{n-1}^{c}\right)^{\prime} \Gamma X_{n}\right)+\left(R_{j}^{c}-\widehat{R}_{n-1}^{c}\right)^{\prime}\left(\widehat{\Gamma}_{n-1}-\Gamma\right) X_{n} h^{\prime}\left(\xi_{j}^{1}\right) \\
& =h\left(Z_{j}^{\prime} X_{n}-\widehat{R}_{n-1}^{c \prime} \Gamma X_{n}\right)+\left(R_{j}^{c}-\widehat{R}_{n-1}^{c}\right)^{\prime}\left(\widehat{\Gamma}_{n-1}-\Gamma\right) X_{n} h^{\prime}\left(\xi_{j}^{1}\right) \\
& =h\left(Z_{j}^{\prime} X_{n}\right)-\widehat{R}_{n-1}^{c \prime} \Gamma X_{n} h^{\prime}\left(\xi_{j}^{2}\right)+\left(R_{j}^{c}-\widehat{R}_{n-1}^{c}\right)^{\prime}\left(\widehat{\Gamma}_{n-1}-\Gamma\right) X_{n} h^{\prime}\left(\xi_{j}^{1}\right) .
\end{aligned}
$$

Since $\widetilde{Z}_{j}-Z_{j}=\left(\widehat{\Gamma}_{n-1}-\Gamma\right) R_{j}^{c}-\widehat{\Gamma}_{n-1} \widehat{R}_{n-1}^{c}$, it follows that:

$$
\begin{aligned}
V_{j} & =\left(\left(\widehat{\Gamma}_{n-1}-\Gamma\right) R_{j}^{c}-\widehat{\Gamma}_{n-1} \widehat{R}_{n-1}^{c}\right) R_{j}^{c \prime} \Gamma\left(X_{n}-\theta\right) h^{\prime}\left(\xi_{j}\right) \\
& +\widehat{\Gamma}_{n-1}\left(R_{j}^{c}-\widehat{R}_{n-1}^{c}\right)\left(-\widehat{R}_{n-1}^{c \prime} \Gamma X_{n} h^{\prime}\left(\xi_{j}^{2}\right)+\left(R_{j}^{c}-\widehat{R}_{n-1}^{c}\right)^{\prime}\left(\widehat{\Gamma}_{n-1}-\Gamma\right) X_{n} h^{\prime}\left(\xi_{j}^{1}\right)\right) .
\end{aligned}
$$

Part 3. For $0<h^{\prime}(x) \leq c$ ( $c=\frac{1}{4}$ for logistic regression, $c=1$ for linear regression), for $j \in I_{n}$ :

$$
\begin{aligned}
\frac{1}{c} E\left[\left\|V_{j}\right\| \mid T_{n}\right] & \leq\left\|\widehat{\Gamma}_{n-1}-\Gamma\right\| E\left[\left\|R^{c}\right\|^{2}\right]\|\Gamma\|\left\|X_{n}-\theta\right\|+\left\|\widehat{\Gamma}_{n-1}\right\|\left\|\widehat{R}_{n-1}^{c}\right\| E\left[\left\|R^{c}\right\|\right]\|\Gamma\|\left\|X_{n}-\theta\right\| \\
& +\left\|\widehat{\Gamma}_{n-1}\right\|\left(E\left[\left\|R^{c}\right\|\right]+\left\|\widehat{R}_{n-1}^{c}\right\|\right)\left\|\widehat{R}_{n-1}^{c}\right\|\|\Gamma\|\left(\left\|X_{n}-\theta\right\|+\|\theta\|\right) \\
& +\frac{1}{2}\left\|\widehat{\Gamma}_{n-1}\right\|\left(E\left[\left\|R^{c}\right\|^{2}\right]+\left\|\widehat{R}_{n-1}^{c}\right\|^{2}\right)\left\|\widehat{\Gamma}_{n-1}-\Gamma\right\|\left(\left\|X_{n}-\theta\right\|+\|\theta\|\right) \text { a.s. }
\end{aligned}
$$

Since $\widehat{\Gamma}_{n-1}$ and $\widehat{R}_{n-1}^{c}$ are $T_{n}$-measurable and converge respectively to $\Gamma$ and 0 , since $\sum_{n=1}^{\infty} a_{n}\left\|\widehat{R}_{n-1}^{c}\right\|<\infty$ and $\sum_{n=1}^{\infty} a_{n}\left\|\widehat{\Gamma}_{n-1}-\Gamma\right\|<\infty$ a.s. by Lemma A.2, it follows that there exist two non-negative $T_{n}$-measurable random variables $D_{n}$ and $E_{n}$ such that for $j \in I_{n}$ :

$$
\left\|E\left[V_{j} \mid T_{n}\right]\right\| \leq D_{n}\left\|X_{n}-\theta\right\|+E_{n}, \sum_{n=1}^{\infty} a_{n} D_{n}<\infty, \sum_{n=1}^{\infty} a_{n} E_{n}<\infty \text { a.s. }
$$

$$
\text { Then } \begin{aligned}
\left|\frac{1}{m_{n}} \sum_{j \in I_{n}}\left\langle X_{n}-\theta, E\left[V_{j} \mid T n\right]\right\rangle\right| & \leq\left\|X_{n}-\theta\right\|\left(D_{n}\left\|X_{n}-\theta\right\|+E_{n}\right) \\
& \leq\left(D_{n}+E_{n}\right)\left\|X_{n}-\theta\right\|^{2}+E_{n} \text { a.s. }
\end{aligned}
$$

Part 4. For $|h(x)| \leq d|x|+e(d=0, e=1$ for logistic regression, $d=1, e=0$ for 
linear regression):

$$
\begin{aligned}
& E\left[\left\|\widetilde{Z}_{j}\left(h\left(\widetilde{Z}_{j}^{\prime} X_{n}\right)-S_{j}\right)\right\|^{2} \mid T_{n}\right] \leq E\left[\left\|\widetilde{Z}_{j}\right\|^{2}\left(d\left\|\widetilde{Z}_{j}\right\|\left(\left\|X_{n}-\theta\right\|+\|\theta\|\right)+e+1\right)^{2} \mid T_{n}\right] \\
& \leq 3 d^{2} E\left[\left\|\widetilde{Z}_{j}\right\|^{4} \mid T_{n}\right]\left(\left\|X_{n}-\theta\right\|^{2}+\|\theta\|^{2}\right)+3(e+1)^{2} E\left[\left\|\widetilde{Z}_{j}\right\|^{2} \mid T_{n}\right] \text { a.s. }
\end{aligned}
$$

For $\gamma \geqslant 1$,

$E\left[\left\|\widetilde{Z}_{j}\right\|^{\gamma} \mid T_{n}\right]=E\left[\left\|\widehat{\Gamma}_{n-1}\left(R_{j}-\widehat{R}_{n-1}\right)\right\|^{\gamma} \mid T_{n}\right] \leq 2^{\gamma-1}\left\|\widehat{\Gamma}_{n-1}\right\|^{\gamma}\left(E\left[\|R\|^{\gamma}\right]+\left\|\widehat{R}_{n-1}\right\|^{\gamma}\right)$ a.s.

Since $\widehat{\Gamma}_{n-1}$ and $\widehat{R}_{n-1}$ are $T_{n}$-measurable and converge respectively to $\Gamma$ and $m$ and since $\sum_{n=1}^{\infty} a_{n}^{2}<\infty$, there exist two non-negative $T_{n}$-measurable random variables $F_{n}$ and $G_{n}$ such that for $j \in I_{n}$ :

$$
\begin{aligned}
& E\left[\left\|\widetilde{Z}_{j}\left(h\left(\widetilde{Z}_{j}^{\prime} X_{n}\right)-S_{j}\right)\right\|^{2} \mid T_{n}\right] \leq F_{n}\left\|X_{n}-\theta\right\|^{2}+G_{n}, \sum_{n=1}^{\infty} a_{n}^{2} F_{n}<\infty, \sum_{n=1}^{\infty} a_{n}^{2} G_{n}<\infty, \\
& \text { then } E\left[\left\|\frac{1}{m_{n}} \sum_{j \in I_{n}} \widetilde{Z}_{j}\left(h\left(\widetilde{Z}_{j}^{\prime} X_{n}\right)-S_{j}\right)\right\|^{2} \mid T_{n}\right] \leq F_{n}\left\|X_{n}-\theta\right\|^{2}+G_{n} \text { a.s. }
\end{aligned}
$$

Part 5. Application of Robbins-Siegmund lemma (Lemma A.1).

By (A1) and (A2):

$$
\begin{aligned}
E\left[\left\|X_{n+1}-\theta\right\|^{2} \mid T_{n}\right] & =\left\|X_{n}-\theta\right\|^{2}-2 a_{n} \frac{1}{m_{n}} \sum_{j \in I_{n}}\left\langle X_{n}-\theta, E\left[V_{j} \mid T_{n}\right]\right\rangle \\
& +a_{n}^{2} E\left[\left\|\frac{1}{m_{n}} \sum_{j \in I_{n}} \widetilde{Z}_{j}\left(h\left(\widetilde{Z}_{j}^{\prime} X_{n}\right)-S_{j}\right)\right\|^{2} \mid T_{n}\right] \\
& -2 a_{n} \frac{1}{m_{n}} \sum_{j \in I_{n}}\left\langle X_{n}-\theta, E\left[Z_{j}\left(h\left(Z_{j}^{\prime} X_{n}\right)-h\left(Z_{j}^{\prime} \theta\right)\right) \mid T_{n}\right]\right\rangle \text { a.s. }
\end{aligned}
$$

Since $h$ is an increasing function:

$$
\left\langle X_{n}-\theta, E\left[Z_{j}\left(h\left(Z_{j}^{\prime} X_{n}\right)-h\left(Z_{j}^{\prime} \theta\right)\right) \mid T n\right]\right\rangle=E\left[\left\|Z_{j}^{\prime}\left(X_{n}-\theta\right)\right\|^{2} h^{\prime}\left(\xi_{j}\right) \mid T_{n}\right] \geqslant 0 \text { a.s. }
$$


By $(\mathrm{A} 5, \mathrm{~A} 6, \mathrm{~A} 7, \mathrm{~A} 8)$ :

$$
\begin{aligned}
E\left[\left\|X_{n+1}-\theta\right\|^{2} \mid T_{n}\right] & \leq\left\|X_{n}-\theta\right\|^{2}\left(1+2 a_{n} D_{n}+2 a_{n} E_{n}+a_{n}^{2} F_{n}\right)+2 a_{n} E_{n}+a_{n}^{2} G_{n} \\
& -2 a_{n} \frac{1}{m_{n}} \sum_{j \in I_{n}} E\left[\left\|Z_{j}^{\prime}\left(X_{n}-\theta\right)\right\|^{2} h^{\prime}\left(\xi_{j}\right) \mid T_{n}\right], \\
\sum_{n=1}^{\infty} a_{n} D_{n} & <\infty, \sum_{n=1}^{\infty} a_{n} E_{n}<\infty, \sum_{n=1}^{\infty} a_{n}^{2} F_{n}<\infty, \sum_{n=1}^{\infty} a_{n}^{2} G_{n}<\infty \text { a.s. }
\end{aligned}
$$

Applying Robbins-Siegmund lemma yields that there exists a non-negative random variable $T$ such that :

$$
\left\|X_{n}-\theta\right\|^{2} \longrightarrow T, \sum_{n=1}^{\infty} a_{n} \frac{1}{m_{n}} \sum_{j \in I_{n}} E\left[\left\|Z_{j}^{\prime}\left(X_{n}-\theta\right)\right\|^{2} h^{\prime}\left(\xi_{j}\right) \mid T_{n}\right]<\infty \text { a.s. }
$$

Part 6. Prove that $T=0$ a.s.

Let $\omega$ be fixed belonging to the intersection of the convergence sets. The writing of $\omega$ will be omitted in the following.

Suppose $T \neq 0$. There exists $0<\epsilon<1$ such that $\epsilon<\left\|X_{n}-\theta\right\|<\frac{1}{\epsilon}$.

Since for $j \in I_{n}, \xi_{j}=\lambda_{j} Z_{j}^{\prime} X_{n}+\left(1-\lambda_{j}\right) Z_{j}^{\prime} \theta=\lambda_{j} Z_{j}^{\prime}\left(X_{n}-\theta\right)+Z_{j}^{\prime} \theta$ with $Z_{j}=\Gamma R_{j}^{c}$, $\left|\xi_{j}\right| \leq\left\|R_{j}^{c}\right\| b$, with $b=\|\Gamma\|\left(\frac{1}{\epsilon}+\|\theta\|\right)$.

For logistic regression, $h^{\prime}(u)=\frac{e^{u}}{\left(1+e^{u}\right)^{2}}$ is an even positive function, decreasing for $u>0$, then $h^{\prime}\left(\xi_{j}\right) \geqslant h^{\prime}\left(\left\|R_{j}^{c}\right\| b\right)>0$. For linear regression, $h^{\prime}(u)=1$.

Let $\lambda_{\min }(A)$ denote the lowest eigenvalue of a matrix $A$; we have for $j \in I_{n}$ :

$$
\begin{aligned}
& E\left[\left\|Z_{j}^{\prime}\left(X_{n}-\theta\right)\right\|^{2} h^{\prime}\left(\xi_{j}\right) \mid T_{n}\right] \geqslant\left(X_{n}-\theta\right)^{\prime} \Gamma E\left[R_{j}^{c} R_{j}^{c \prime} h^{\prime}\left(\left\|R_{j}^{c}\right\| b\right) \mid T_{n}\right] \Gamma\left(X_{n}-\theta\right) \\
& \geqslant \lambda_{\min }\left(E\left[R^{c} R^{c \prime} h^{\prime}\left(\left\|R_{j}^{c}\right\| b\right)\right]\right)\left\|\Gamma\left(X_{n}-\theta\right)\right\|^{2} \geqslant \lambda_{\min }\left(E\left[R^{c} R^{c \prime} h^{\prime}\left(\left\|R_{j}^{c}\right\| b\right)\right]\right)\left(\lambda_{\min }(\Gamma)\right)^{2} \epsilon^{2} .
\end{aligned}
$$

The symmetric matrix $E\left[R^{c} R^{c \prime} h^{\prime}\left(\left\|R_{j}^{c}\right\| b\right)\right]$ is positive definite since by H1a there is no linear relationship between the components of $R^{c}$, thus between the components of $R^{c}\left(h^{\prime}\left(\left\|R_{j}^{c}\right\| b\right)\right)^{\frac{1}{2}}$; its lowest eigenvalue is strictly positive. By $\mathrm{H} 2$, it follows that:

$$
\begin{aligned}
& \sum_{n=1}^{\infty} a_{n} \frac{1}{m_{n}} \sum_{j \in I_{n}} E\left[\left\|Z_{j}^{\prime}\left(X_{n}-\theta\right)\right\|^{2} h^{\prime}\left(\xi_{j}\right) \mid T_{n}\right] \\
& \geqslant \lambda_{\min }\left(E\left[R^{c} R^{c \prime} h^{\prime}\left(\left\|R_{j}^{c}\right\| b\right)\right]\right)\left(\lambda_{\min }(\Gamma)\right)^{2} \epsilon^{2} \sum_{n=1}^{\infty} a_{n}=\infty
\end{aligned}
$$

This is a contradiction since $\omega$ belongs to the convergence set of this series. Thus $T=0$. We deduce immediately the convergence of $\left(\bar{X}_{n}\right)$ to $\theta$. 


\section{Appendix B. Additional results regarding HOSPHF30D}

For HOSPHF30D, all processes AS.P. have a criterion value lower than 0.05 after $300 N$ observations used (Table B1).

Table B1. Evolution of the relative norms for HOSPHF30D after $100 N$ observations used

\begin{tabular}{lccccc}
\hline Process & $100 N$ & $200 N$ & $300 N$ & $400 N$ & $500 N$ \\
\hline CS1V & 0.088 & $0.045^{*}$ & $0.034^{*}$ & $0.021^{*}$ & $0.023^{*}$ \\
\hline CS10V & 0.260 & 0.178 & 0.115 & 0.098 & 0.088 \\
\hline CS100V & 0.629 & 0.499 & 0.406 & 0.357 & 0.322 \\
\hline AS1P50 & 0.064 & $0.049^{*}$ & $0.013^{*}$ & $0.016^{*}$ & $0.009^{*}$ \\
\hline AS10P50 & 0.060 & 0.051 & $0.013^{*}$ & $0.016^{*}$ & $0.010^{*}$ \\
\hline AS100P50 & 0.067 & 0.056 & $0.019^{*}$ & $0.018^{*}$ & $0.013^{*}$ \\
\hline AS1P100 & 0.062 & $0.045^{*}$ & $0.013^{*}$ & $0.016^{*}$ & $0.009^{*}$ \\
\hline AS10P100 & 0.060 & $0.048^{*}$ & $0.012^{*}$ & $0.015^{*}$ & $0.009^{*}$ \\
\hline AS100P100 & 0.065 & 0.055 & $0.015^{*}$ & $0.016^{*}$ & $0.011^{*}$ \\
\hline AS1P200 & 0.057 & $0.040^{*}$ & $0.015^{*}$ & $0.015^{*}$ & $0.011^{*}$ \\
\hline AS10P200 & 0.059 & $0.045^{*}$ & $0.012^{*}$ & $0.015^{*}$ & $0.008^{*}$ \\
\hline AS100P200 & 0.066 & 0.053 & $0.013^{*}$ & $0.016^{*}$ & $0.010^{*}$ \\
\hline
\end{tabular}

* denotes a criterion value $<0.05$.

For HOSPHF30D, processes AS10P. and AS100P. have a criterion value lower than 0.05 at $120 \mathrm{~s}$ (Table B2).

Table B2. Evolution of the relative norms for HOSPHF30D after 60 s of processing time

\begin{tabular}{lcccc}
\hline Processus & 60s & 120s & 180s & 240s \\
\hline CS1V & 0.153 & 0.161 & 0.125 & 0.064 \\
\hline CS10V & 0.228 & 0.158 & 0.103 & 0.087 \\
\hline CS100V & 0.326 & 0.231 & 0.167 & 0.141 \\
\hline AS1P50 & 0.095 & 0.088 & 0.064 & 0.073 \\
\hline AS10P50 & 0.053 & $0.034^{*}$ & $0.018^{*}$ & $0.010^{*}$ \\
\hline AS100P50 & $0.014^{*}$ & $0.010^{*}$ & $0.012^{*}$ & $0.014^{*}$ \\
\hline AS1P100 & 0.087 & 0.086 & 0.062 & 0.063 \\
\hline AS10P100 & $0.050^{*}$ & $0.032^{*}$ & $0.017^{*}$ & $0.009^{*}$ \\
\hline AS100P100 & $0.011^{*}$ & $0.009^{*}$ & $0.014^{*}$ & $0.015^{*}$ \\
\hline AS1P200 & 0.112 & 0.081 & 0.062 & $0.050^{*}$ \\
\hline AS10P200 & $0.049^{*}$ & $0.027^{*}$ & $0.010^{*}$ & $0.009^{*}$ \\
\hline
\end{tabular}

* denotes a criterion value $<0.05$. 